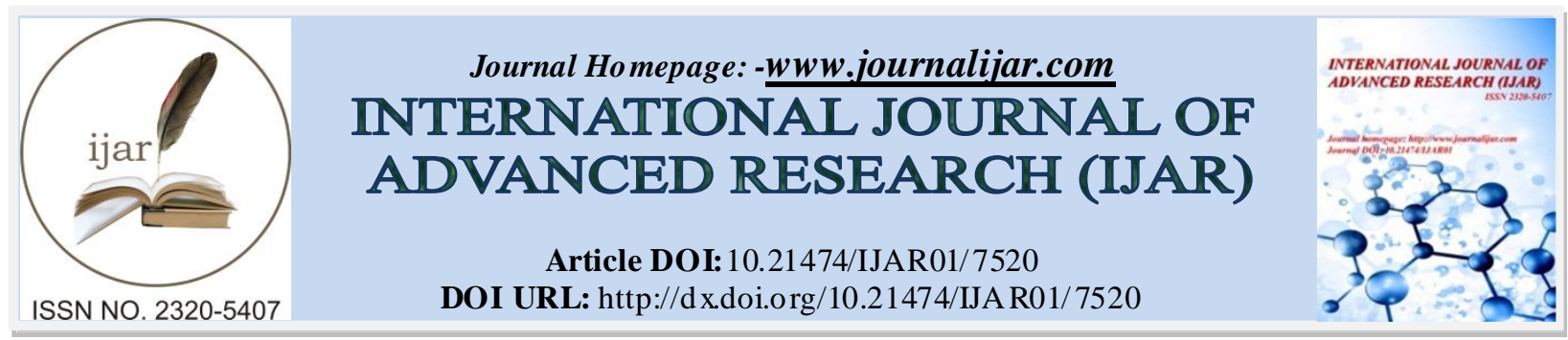

RESEARCH ARTICLE

\title{
EVALUATION OF LIQUORICE GLYCYRRHIZA GLABRA L. ROOT POWDER AS A NEW ADSORBENT IN THE REMOVAL OF TEXTILE DYES IN AQUEOUS MEDIUM.
}

AraceliVerônica F. N. Ribeiro ${ }^{1}$, André Romero da Silva ${ }^{1}$, Paulo Henrique dos Santos Silvares ${ }^{2}$, Amanda de Oliveira Loiola ${ }^{2}$, Flávio Cunha Monteiro ${ }^{2}$, Madson de Godoi Pereira ${ }^{3}$ and $^{*}$ JoselitoNar dy Ribeiro $^{2}$.

1. Instituto Federal do Espírito Santo, Vila Velha - ES, Brazil.

2. GIBBA-Universidade Federal do Espírito Santo, Maruípe, Vitória - ES, Brazil.

3. Universidade do Estado da Bahia-UNEB, Salvador-BA, Brazil.

\section{Manuscript Info}

(..........................

Manuscript History

Received: 06 June 2018

Final Accepted: 08 July 2018

Published: August 2018

Keywords:-

adsorption, congo red, indigo blue, Glycyrrhizaglabra L. Root powder.

\begin{abstract}
The liquorice Glycyrrhizaglabra L. root powder (GGRP) was evaluated to remove the two textile dyes in water. The physical-chemical analyzes using the scanning electron microscope and infrared spectroscopy showed that this material has irregular surface and chemical groups capable of retaining chemical pollutants in aqueous medium. In addition, our results demonstrated that it was possible to obtain a satisfactory adsorption percentage of the indigo blue (IB) and congo red dye (CR) when used $2.0 \mathrm{~g}$ of GGRP and stirring time at 5 minutes. The maximu m adsorption capacity for IB $\left(1.696 \mathrm{mg} \cdot \mathrm{g}^{-1}\right)$ and CR $\left(0.768 \mathrm{mg} . \mathrm{g}^{-1}\right)$ obtained through the Langmuir Mathematical Model suggest GGRP as an possible adsorbent for removal both textile dyes. This conclusion could be sustained through bacth column experiments where GGRP powder was more efficient than activated carbon (13.5 \pm $2.1 \%$ for IB and $16.9 \pm 3.2 \%$ for CR) in the removal of IB $(98.2 \pm 1.2$ $\%)$ and CR $(82.1 \pm 3.5 \%)$. Finally, th is work provided a new alternative for the use of GGRP an environ mental decontaminant and not only for medicinal use.
\end{abstract}

Copy Right, IJAR, 2018,. All rights reserved.

\section{Introduction:-}

A large number of dyes have been synthesized in the last hundred years (Guaratini\&Zanoni, 2000). The high demand for these chemicals, mainly textile dyes, shows the high textile industry growth and consequently a considerable increase in the discharge of textile effluents contaminated with several types of dyes. This is worrying since most of these effluents, in many countries, are not treated or the treatments are not satisfactory (Mathur et al., 2005). The textile effluents have strong color due to the non-fixing of excessive dye added to fibers during tinting process (Kunz et al., 2002). Every year tons of textile dyes are discarded in the aquatic environment in countries such as Brazil and India (Guaratini\&Zanoni, 2000; Mathur et al., 2005). Most discarded dyes have stable chemical structures and, therefore, are difficult to biodegrade (Forgacs et al., 2004). For example, congo red is an azo dye (R$\mathrm{N}=\mathrm{N}-\mathrm{R}$ ) (figure 1) considerably very stable in aqueous medium. This organic compound denominated the disodium salt of 3,3'-([1,1'-biphenyl]-4,4'-diyl)bis(4-aminonaphthalene-1-sulfonic acid) is very soluble in water and largely utilized for tinting of cellu lose fiber such as cotton and viscose (Guaratini\&Zanoni, 2000). Another dye widely used in the textile industry is indigo (figure 2), predominantly blue. This dye was extracted from plants, principally

Corres ponding Author:-AraceliVerônica F. N. Ribeiro.

Address:-Instituto Federal do Espírito Santo, Vila Velha - ES, Brazil. 
Indigoferatinctoria. However, its industrial synthes is is the most used today. Indigo blue is commonly used in textile industry for the dyeing principally of common cotton and jeans (Baig, 2012).

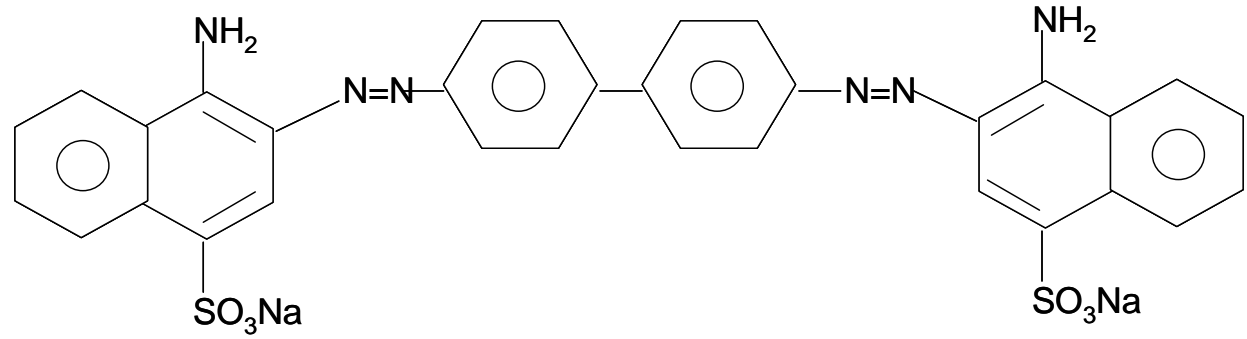

Figure 1:- Chemical structure of congo red

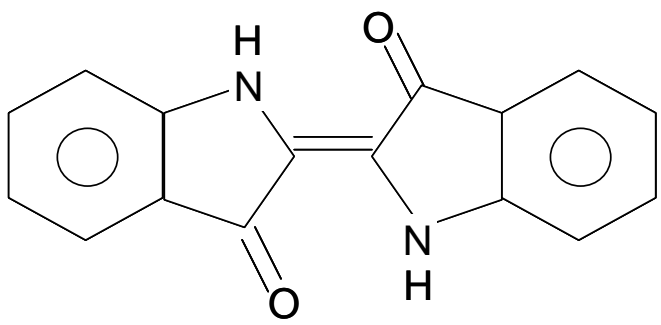

Figure 2:- Che mical structure of indigo blue

One of the main problems of dyes comes from their accumulation and bioavailability. The excess of these organic compounds can be dangerous to the environment and human health (Guaratini\&Zanoni, 2000). Textile dyes can affect the photosynthesis process, as well as cause respiratory problems, skin sensitivity, mutagenicity and others (Guaratini\&Zanoni, 2000; Mathur et al., 2005; Raymundo et al., 2010). Therefore, studies for the development of technologies for the removal of textile dyes in water are very important. Some processes are being evaluated such as biodegradation (Adedayo et al., 2004), chemical degradation (Chen et al., 2018), photochemical degradation (Pagano et al., 2018) and adsorption processes using synthetic (Farrokhi-Rad et al., 2018) and natural adsorbents (Maghri et al., 2012).

Adsorption is a process of accumulation and selective concentration of one or more adsorbates on solid surfaces of adsorbents. The adsorbate may be present in gases (Mohammadi-Moghadam et al., 2013) or liquids (Maghri et al., 2012). In relation to the treatment of effluents the adsorbates are pollutants such as heavy metals (Jordão et al., 2010), pharmaceuticals (Ribeiro et al., 2016) textile dyes (Pereira et al., 2009) and others (Amin et al., 2015). Adsorption using natural adsorbents has been one of the most studied due to considerable availability and low cost (Ramakrishna \&Viraraghavan, 1997). So me examples can be cited as: sugar cane bagas se was evaluated to removal of congo red dye (Raymundo et al., 2010) and malachite green dye (Tahir et al., 2016), vermicompost was able to removal of crystal violet and methylene blue in water (Pereira et al., 2009), wood sawdust was studied to removal of textile dyes (SahMoune\&Yeddou, 2016), and banana peel powder was studied to removal of anionic dyes from aqueous solutions (Munagapat et al., 2018).

The liquoriceGlycyrrhizaglabra L. root is more related to its medicinal properties and culinary or flavour potential (Fenwick, 1990). The use of Glycyrrhizaglabra L. root powder (GGRP) as an adsorbent to removal pollutants is almost absent in the literature. In 2013 researchers reported the application of GlycyrrhizaglabraL. root as a novel adsorbent in the removal of toluene vapors (Mohammadi-Moghadam et al., 2013). However, studies on GGRP as an adsorbent for pollutants in aqueous media are scarce. Therefore, this work aimed to evaluate the ability of GGRP as adsorbent for the removal of textile dyes in water. The congo red (CR) and indigo blue (IB) dyes were used as pollutants in this study. For this evaluation we performed studies on the physico-chemical characteristics of GGRP, influence of the GGRP mass in adsorptive process, and influence of stir time between CR or IB and GGRP. Subsequently, the maximum adsorptive capacities (MAC) of GGRP for CR and IB were determined. Finally the efficiency of GGRP was compared with activated carbon for the removal of both dyes in batch column experiments. 


\section{Materials and methods:- \\ Materials:-}

The liquoriceGlycyrrhizaglabra L. root powder (GGRP) was obtained from EmpórioFigueiras (Santo André-SP, Brazil), The activated carbon (AC) was obtained from Casa das Químicas (Flores da Cunha-RS, Brazil). Congo red dye (CR) (figure. 1) was purchased from Vetec Company (Duque de Caxias-RJ, Brazil). Indigo Blue (IB) (figure. 2) was purchased from Tupy Industry (São Paulo-SP, Brazil). The following equipment was used in laboratory tests: laboratory oven (Quimis Q-317 B model), particle size sieves (Granutest), Analytical balance (Shimadzu A Y 220 model), sputter coater (Shimadzu, IC-50 Ion Coater model), scanning electron microscope (Shimadzu, SSX 550 model), infrared spectrophotometer (Perkin-Elmer Spectru -100 model), pHmeter (PHTEK), magnetic stirrer (Warmnest), UV/Vis spectrophotometer (Biospetro SP-220 model), microcentrifuge 13,000 rpm (Evlab), and vacuum filtration pump (Pris matec). Several common laboratory glassware was used such as glass columns, beckers, erle meyers, kitassato, burette, glass funnel and others.

\section{Methods:- \\ Preparation of the ads or bent:-}

The liquorice Glycyrrhizaglabra L. root powder (figure 3) was washed with distilled water $\mathrm{pH} 6.63$ and $\mathrm{pH}$ adjusted to $7.01 \mathrm{using}$ sodium hydroxide $0.01 \mathrm{M}$. Subsequently the adsorbent material was dried in a laboratory over for 15 hrs at $40^{\circ} \mathrm{C}$. Finally the liquorice GGRPwas sieved to obtain particles of size smaller than $1.19 \mathrm{~mm}$. The material was finally stored in hermetically sealed containers for use in the later stages.

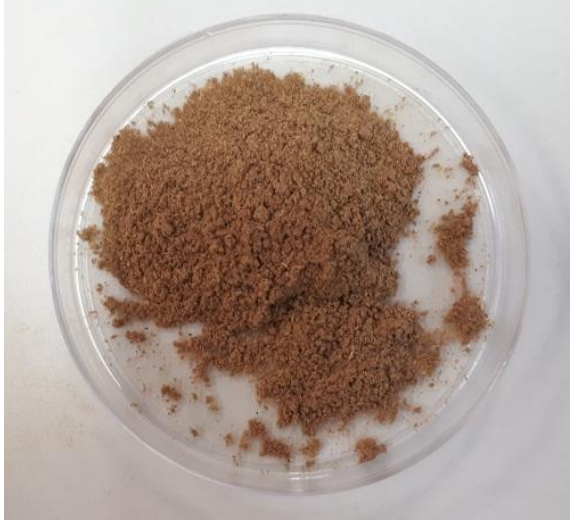

Figure 3:- The liquorice Glycyrrhizaglabra L. root powder

\section{Scanning electron microscopy:-}

In order to know the characteristics of the GGRP surface, an analysis was performed using scanning electron microscopy (SEM). For this, a small sample of GGRP was immersed in a thin layer of gold using the spray coating. Then the surface of this sample was visualized in SEM (electron beam of $10 \mathrm{kV}$ ) as described in Ribeiro et al. (2018).

\section{Infrared s pectr os copy:-}

To describe the chemical groups present in GGRP, an infrared spectroscopy (IR) analysis was performed as described in Ribeiro et al. (2016). This methodology was performed by Fourier transform IR spectroscopy using $\mathrm{KBr}$ discs to prepare the GGRP samples. The spectral range varied from 4000 to $500 \mathrm{~cm}^{-1}$. This stage aims to determine if the chemical groups of GGRP are compatible with the chemical structures of CR and IB for the occurrence of interactions favorable to the adsorptive process.

\section{Stirring time:-}

The $50 \mathrm{ml}$ of aqueous solutions ( $\mathrm{pH} 7.01$ ) containing $87 \mathrm{mg} / \mathrm{L}$ of IB or $25 \mathrm{mg} / \mathrm{L}$ of CR were magnetically stirred $(1000 \mathrm{rpm})$ at different times (minutes) at $25 \pm 1^{\circ} \mathrm{C}$. After this procedure, the solutions were filtered at vacuum for retention of the adsorbent using quantitative paper filter (Unifil). The supernatants were centrifuged at 13,000 rpm and analyzed in a spectrophotometer at $500 \mathrm{~nm}$ to $\mathrm{CR}$ and $573 \mathrm{~nm}$ to IB to evaluate the amount (\%) of dye retained in the adsorbent (figure 4). This step had as objective to evaluate the influence of the contact time between dyes and adsorbent in the adsorption process [6]. The te mperature, stirring speed, and pH were optimized in our laboratory. 
(A)

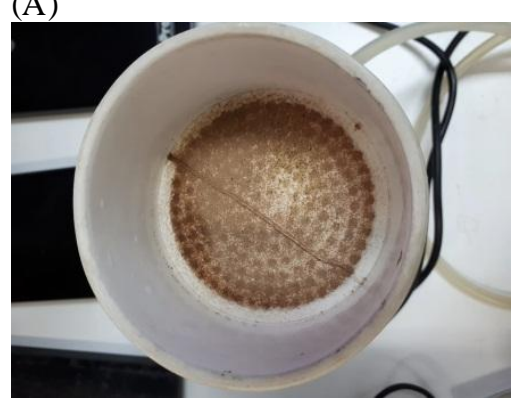

Figure 4:-The aqueous solutions filtered
(B)

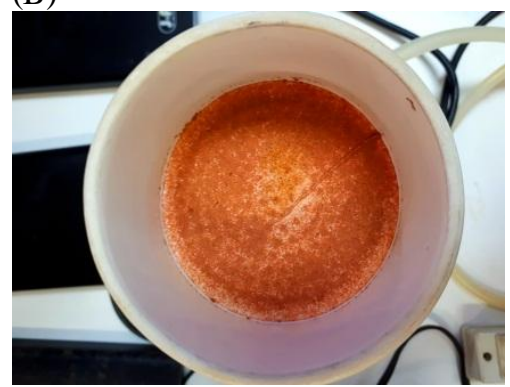

(C)

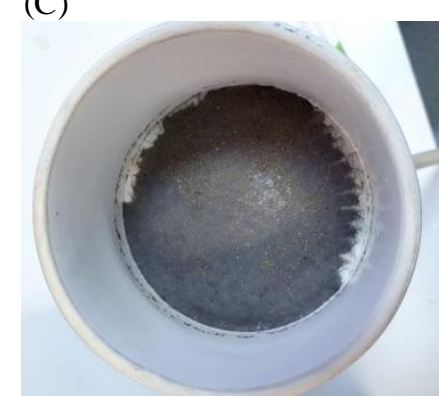

in the presence of CR (B) and IB (C).

Ads orbent mass:-

Using the conditions previously described and optimized agitation time in the previous step (5 minutes) the aqueous solutions $(50 \mathrm{ml})(\mathrm{pH} 7.01)$ containing IB $(87 \mathrm{mg} / \mathrm{L})$ or CR $(25 \mathrm{mg} / \mathrm{L})$ we re magnetically stirred at diffe rent GGRP mass ( $\mathrm{g}$ ). After this procedure, the solutions were filtered at vacuum for retention of the adsorbent using quantitative paper filter (Unifil). The supernatants were centrifuged at 13,000 rpm and analyzed in a spectrophotometer at 500 $\mathrm{nm}$ to $\mathrm{CR}$ and $573 \mathrm{~nm}$ to IB to evaluate the amount $(\%)$ of dye retained in the different adsorbent mass. The finality of this step was to evaluate the influence of the adsorbent mass in the adsorption equilibrium process.

\section{Maxi mum ads or ption capacity:-}

The equations 1 and 2 were fundamental to determine the values of maximum adsorptive capacities (MAC) of GGRP to CR and IB. These values represent the maximu m amounts of these dyes which can be adsorbed by $1 \mathrm{~g}$ of GGRP. A high MAC value reveals that the ads orptive process is efficient:

$q=a \times b \times E q c \times(1+a \times E q c)^{-1}$

$E q c / q=(a x b)^{-1}+(b)^{-1} \times E q c$

where $q$ is the quantity of adsorbed dye in GGRP ( $\mathrm{mg} / \mathrm{g})$, a is a constant related to adsorption energy (L/mg), $b$ is the maximum dye adsorption capacity of the GGRP ( $\mathrm{mg} / \mathrm{g}$ ), and $E q c$ is the equilibrium dye concentration (mg/L). Equation 2 is obtained by linearizing equation 1, which permits exact calculations of $b$ values. From these equations, it was possible to calculate the maximum adsorption capacities ( $b^{-1}=$ MAC) of GGRP to CR and IB. To obtain these MAC values, experiments were performed in which aqueous solutions (50 ml) of IB or CR at different and increasing concentrations $(\mathrm{mg} / \mathrm{L})$ were stirred in the conditions previously established (Stirring time: 5.0 minutes; stirring speed: $1,000 \mathrm{rpm}$; adsorbent mass: $2.0 \mathrm{~g}$; $\mathrm{pH} 7.01$; temperature: $25 \pm 1^{\circ} \mathrm{C}$ ). After this procedure, the solutions were filtered at vacuum for retention of the adsorbent using quantitative paper filter (Unifil). The supernatants were centrifuged at $13,000 \mathrm{rpm}$ and analyzed in a spectrophotometer at $500 \mathrm{~nm}$ to $\mathrm{CR}$ and $573 \mathrm{~nm}$ to IB to evaluate the amount (\%) of dye retained in the different dyes concentrations.

\section{Dyes removal in glass columns:-}

To test the adsorbent under almost real conditions, water samples from Espírito Santo Brazilian State Sanitation Company were enriched with $25 \mathrm{mg} / \mathrm{L}$ CR or $87 \mathrm{mg} / \mathrm{L} \mathrm{IB}$. The $\mathrm{pH}$ of these samples was around 7.0 with standardized values for colour (4 mg PT-Co/L; Hazem Unity), total dis solved solids (160 mg/L) and turbidity (2.17 NTU; Nephelometric Turbidity Unity). Subsequently, three glass columns $(38$ x $3 \mathrm{~cm})$ were filled with $5.0 \mathrm{~g}$ of activated carbon (AC) in $3.0 \pm 0.4 \mathrm{~cm}$ of height and $12 \mathrm{~g}$ of gravel in $5.0 \pm 0.8 \mathrm{~cm}$ of height. Another three glass columns were equally filled, but with the addition of $5.0 \mathrm{~g}$ of GGRP in $4.5 \pm 0.6 \mathrm{~cm}$ height. Then $50 \mathrm{ml}$ of dye (CR or IB) aqueous solutions were percolated through the columns (figure 5). The flow rate used was $5.0 \mathrm{~mL} / \mathrm{min}$. for all columns (optimized in our laboratory). The filtered solutions were centrifuged at 13,000 rpm and analyzed in a spectrophotometer at $500 \mathrm{~nm}$ to $\mathrm{CR}$ and $573 \mathrm{~nm}$ to IB to evaluate the amount $(\%)$ of dye retained in the column (Ribeiro ${ }^{\text {e }}$ et al., 2016). 


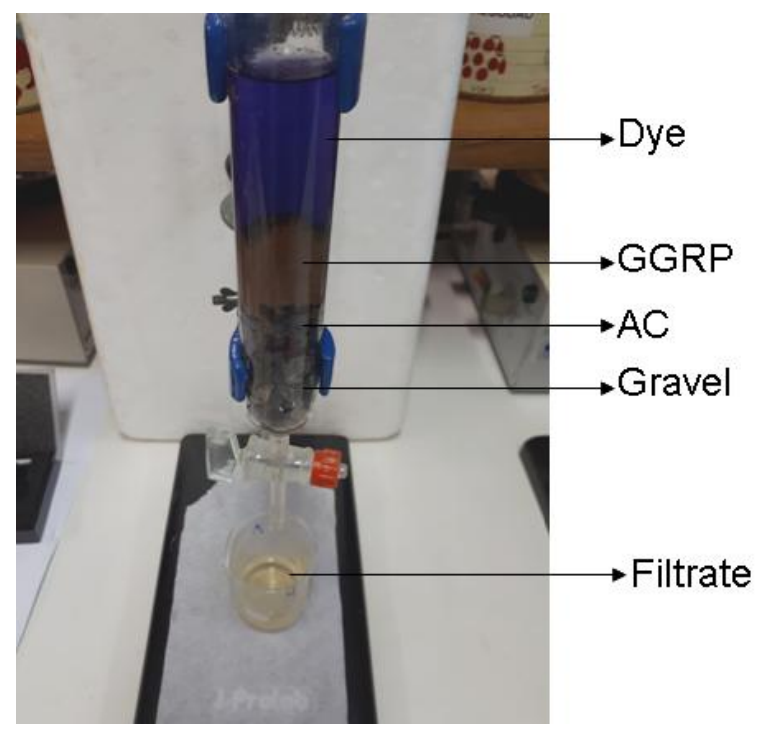

Figure 5:-Glas s column to filtration of aqueous solutions containing CR or IB in the presence of GGRP, AC and gravel. The figure of the colu mn in the absence of GGRP does not appear.

\section{Results and Discussion:-}

Scanning electron microscopy:-

The SEM analysis revealed that the GGRP surface is not homogeneous (figure 6). Surface with irregularities are crucial for efficient adsorption and have been observed in natural adsorbents studied for the removal not only of dyes (Pereira et al., 2009; Ribeiro et al., 2018) but also of other pollutants (Ribeiro et al., 2014; Ribeiro et al., 2016; Ribeiro ${ }^{b}$ et al., 2016).

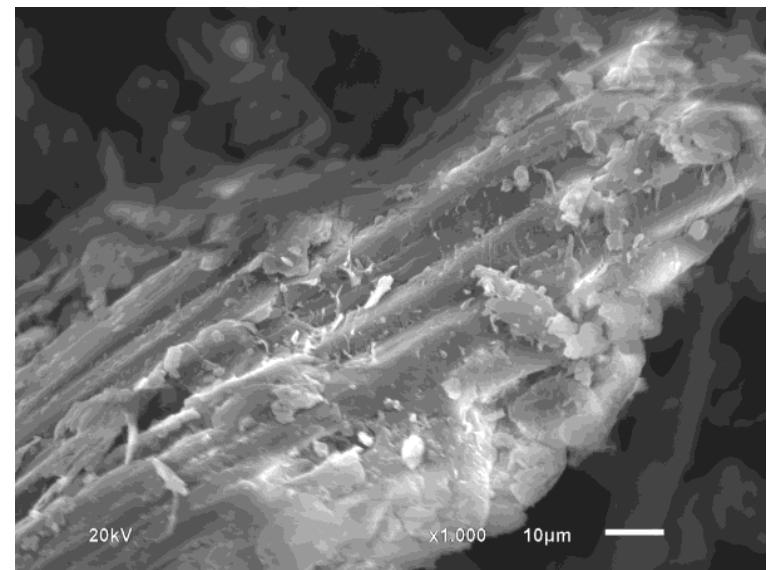

Figure 5:- Scanning electron microscopy of Glycyrrhizaglabra L. root powder with magnifications of $1000 \mathrm{x}$

Pereira et al. (2009) verified that not homogeneous surface of vermicompost was important for cationic dyes removal in aqueous medium. Ribeiro et al. (2018) and Ribeiro et al. (2016) showed that heterogeneous surface of wood sawdust powder and green mesocarp coconut could be related to the efficient adsorption of CR and drugs respectively. Additionally Ribeiro et al. (2014) proposed that the irregular surface of sugarcane bagasse could be one of the factors responsible for the efficiency in the removal of tetracycline in water. Finally Pereira et al. (2012) showed that SEM and other techniques revealed desirable adsorption of heavy metals by sediments, clay and total organic matter $(\%, \mathrm{~m} / \mathrm{m})$ in JacuipeRiver's estuary (northeastern Brazil).

\section{Infrared s pectros copy:-}

The chemical composition of liquorice root is complex (Fenwick, 1990). Approximately 5 to $24 \%$ of its composition is due to a biologically active triterpene glycoside (glycyrrhizin), 30\% starch, and 3-16\% sugar (Duke, 1985) such as glucose, maltose, sucrose, and fructose. It was also noted the presence of dextrins, pentosans, lignin, and others 
(Fenwick, 1990). One can not fail to mention the presence of others several chemical co mpounds such as flavonoids, is oflavonoids, chalcones, coumarins (Lutomski, 1983), aminoacids, and others low molecular weight compounds (Fenwick, 1990). The presence of this diversity of chemical compounds gives the aqueous extracts of the liquorice many therapeutic and others properties (Fenwick, 1990). The IR analysis of GGRP (figure 6) shows similar characteristics to the spectra of other adsorbents of vegetable origin (Ribeiro et al., 2014; Ribeiro et al., 2018). The presence of spectroscopic bands related to some chemical groups possibly is due the occurrence of biomolecules such as cellulose, hemicellu lose, lignin and others (Ribeiro b et al., 2016; Ribeiro et al., 2018). The IR of GGRP (figure 6) revealed the presence of O-H group $\left(3307 \mathrm{~cm}^{-1}\right.$ ) probably from the alcohol and other compounds with axial deformation due to intermolecular hydrogen bonds. The C-H from methyl group $\left(2920 \mathrm{~cm}^{-1}\right) \mathrm{and} \mathrm{probably}^{-\mathrm{m}}$ $\mathrm{C}=\mathrm{O}$ (carbonyl) in $1620 \mathrm{~cm}^{-1}$, also were revealed. Additionally the presence of $\mathrm{C}-\mathrm{O}$ group was also detected at approximately $1023 \mathrm{~cm}^{-1}$, probably due to axial deformation present in C-O-C system (Wong, 2015). Most of these chemical groups must belong to insoluble biomolecules, since the process of washing the powder with water should remove most of the soluble compounds. The presence of these and other chemical groups is to fundamental relevance to the possible chemical interactions between the dyes and adsorbent (Pereira et al., 2009).

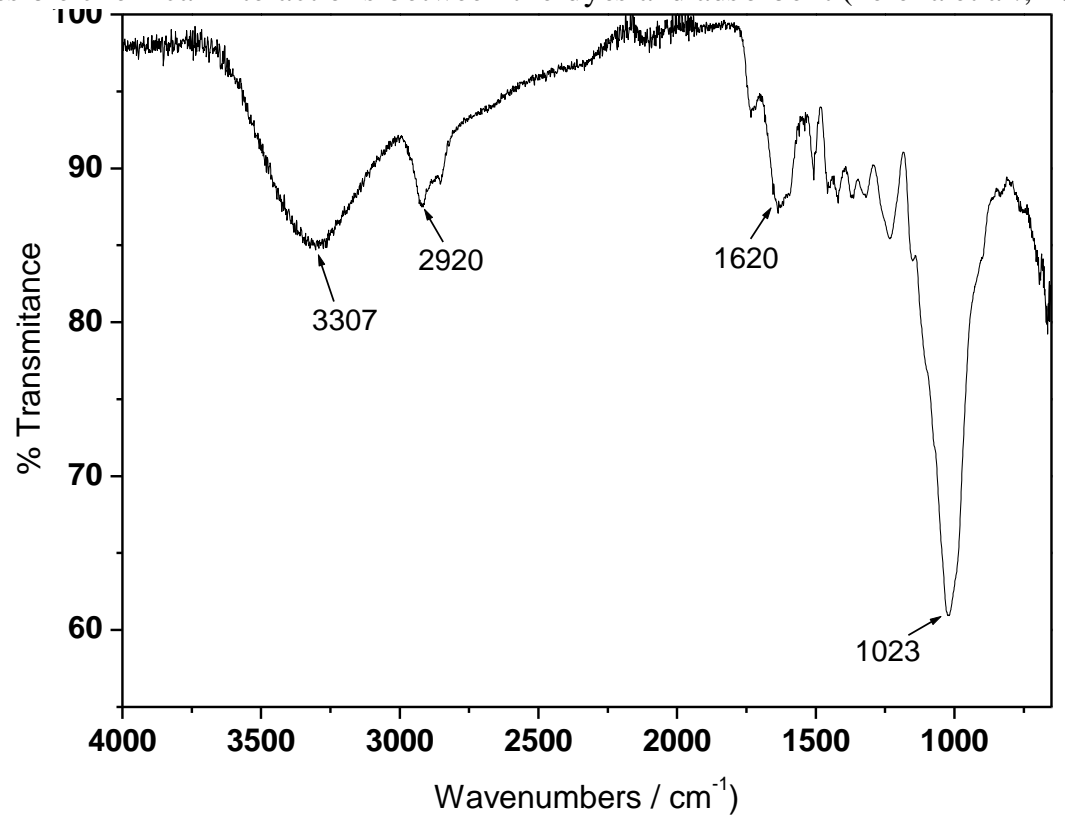

Figure 6:-IR spectro of ofGlycyrrhizaglabra root powder

\section{Stirring time:-}

The time of contact between adsorbent and adsorbate is important to determine in which time equilibrium will be established in the adsorption percentage. His step determines one of the parameters required in the test that establishes conditions for calculating the MAC value. Raymundo et al. (2010) demonstrated the percentage of CR adsorption by sugarcane bagasse increases according to the stirring time. However, after a certain time, the adsorption decreases suggesting an adsorption process accompanied by desorption. A similar result was observed by Ribeiro et al. (2016) when evaluating acetylsalicylicacidretention by bananapeel. However Ribeiro et al. (2018) demonstrated that the adsorption of CR by wood sawdust powder from Corymbiacitriodoraincreases up to 5 minutes and fromthis point remains constant. A similarresultwasfound in thisstudy. For both CR (figure 7) and IB (figure 8) the maximu $\mathrm{m}$ adsorption time wasin 5 minutes remaining constant fromthis point. 


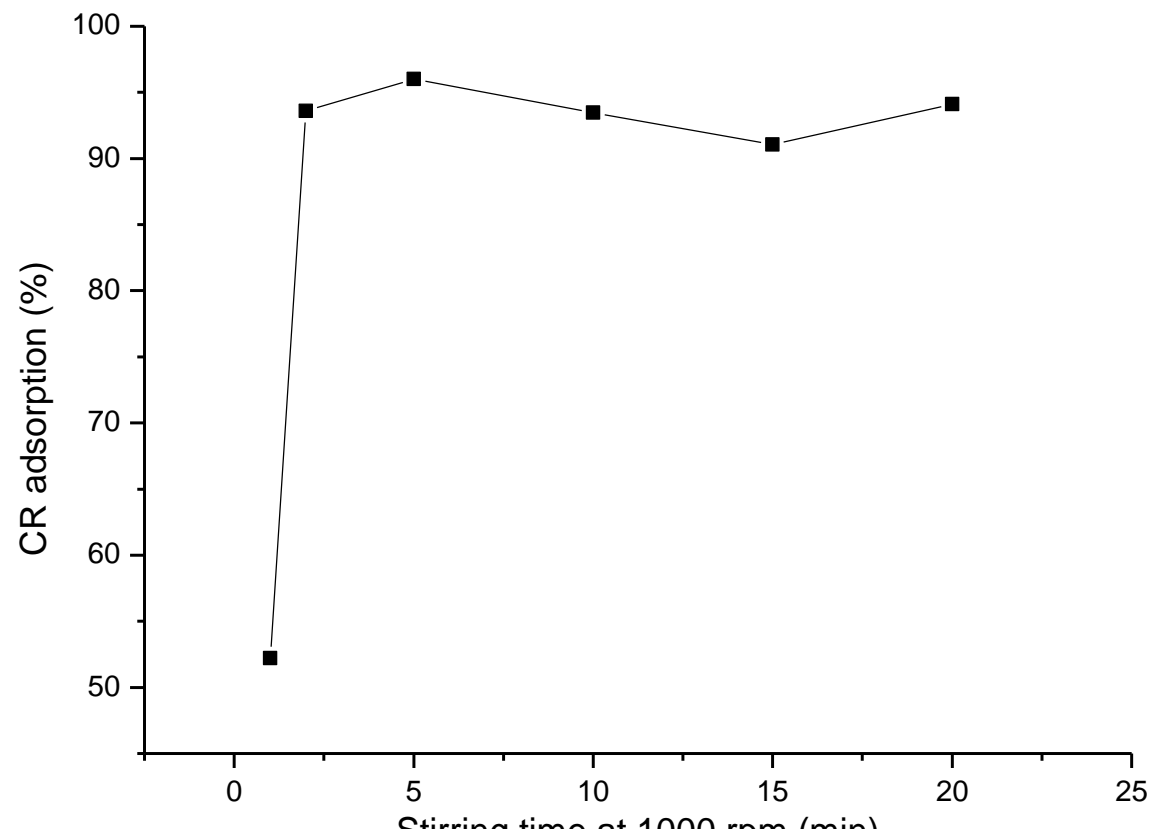

Figure 7:- In fluence of magnetic stirring time $(1000 \mathrm{rpm})$ in CR adsorption in the presence of GGRP

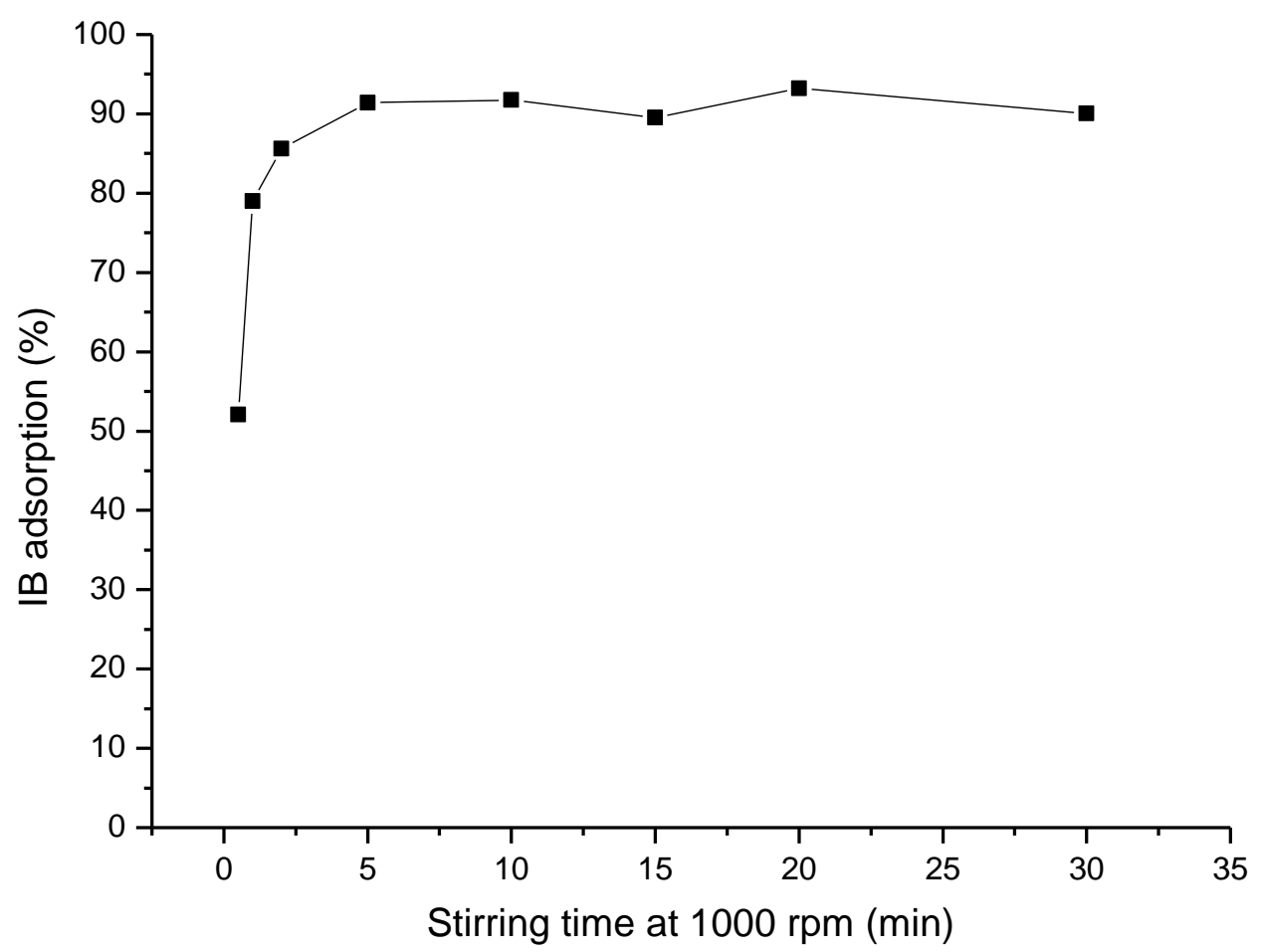

Figure 8:- In fluence of magnetic stirring time $(1000 \mathrm{rpm})$ in IB adsorption in the presence of GGRP

Adsorbent mass :-

Ribeiro et al. (2018) evaluated the influence of the mass of the wood sawdust powder adsorbent from Corymbiacitriodora (WSPECC)to remove the CR in aqueous medium. The maximum percentage of CR adsorption 
was obtained in the presence of $1.5 \mathrm{~g}$ of WSPECC. From this mass the percentage of adsorption remained constant and reached equilibrium. Ray mundo et al. (2010) demonstrated that the increase in the mass of sugarcane bagasse powder increased the percentage of CR adsorption. The results indicated that the maximum adsorption (88\%) occurred with the use of $10 \mathrm{~g}$ of adsorbent and then remained constant reaching equilibrium. Pereira et al. (2009) demonstrated that crystal violet dye was efficiently retained on vermicompost $(99.9 \pm 0.07 \%)$ when $4.8 \mathrm{~g}$ of the referred adsorbent was employed. Moreover, a vermicompost mass of $0.6 \mathrm{~g}$ was necessary to retain $99.0 \pm 0.1 \%$ of methylene blue dye. According to the authors this expressive difference in the adsorbent mass can be attributed to steric hindrance, since molecules of methylene blue are smaller than those of crystal violet. It is suggested that the increase in the amount of adsorption with the increase of the mass of the adsorbent occurs due to the increase in the availability of adsorptive sites, until the process reaches the adsorption equilibrium. Similar results were observed in the present study. For both CR (figure 9) and IB (figure 10) the increase in GGRP mass resulted in an increase in the adsorption percentage of the dyes. Interestingly for both the maximum adsorption was established with the use of $2.0 \mathrm{~g}$ of GGRP. This may be related to similar aspects in the interactions of both dyes with the adsorbent.

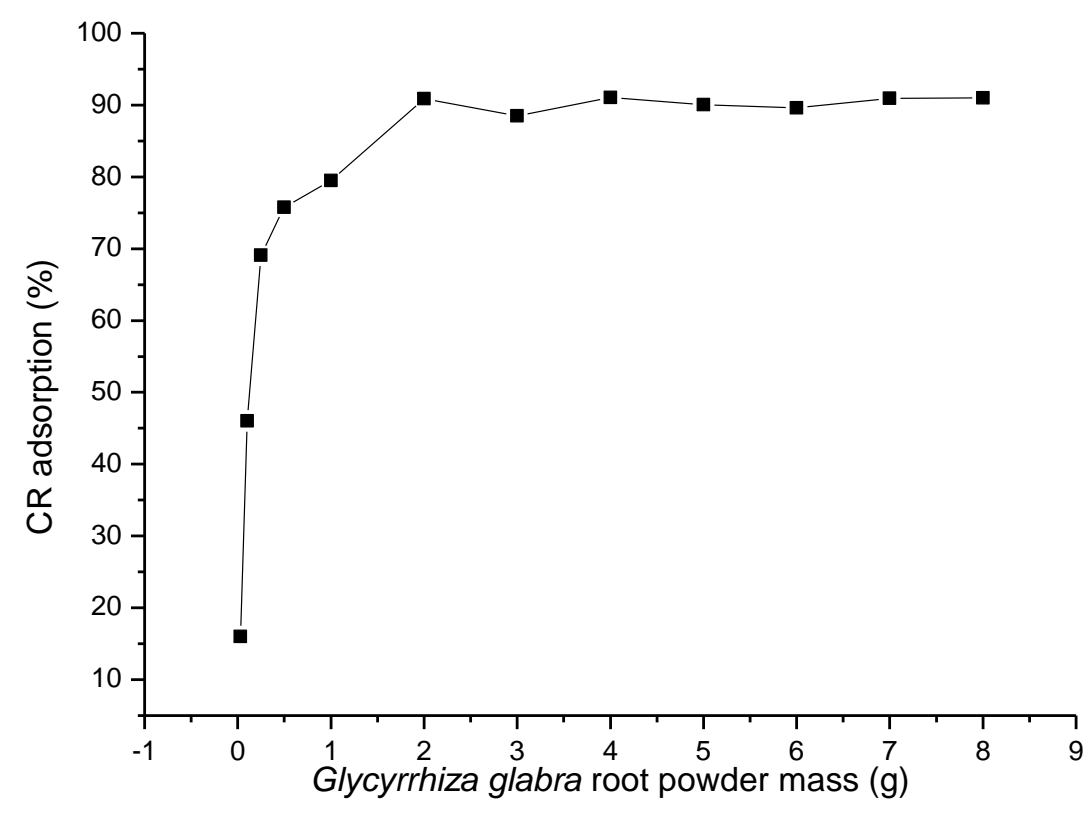

Figure 9:- In fluence of adsorbent mass in CR adsorption 


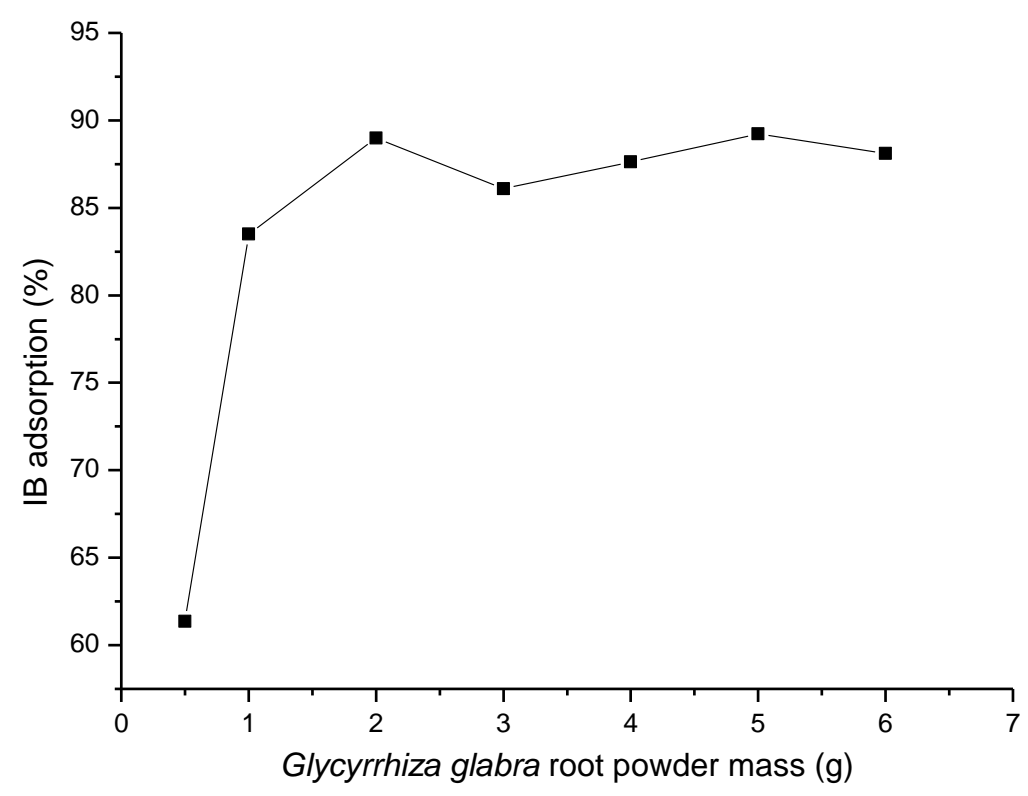

Figure 10:- Influence of adsorbent mass in IB adsorption

\section{Maximum ads or ption capacity: -}

Through the data obtained in this step the adsorption isotherms for CR (figure 11) and IB (figure 12) were elaborated. After linearization of these isotherms (figures 13 and 14) it was possible to calculate the MAC values for CR (0.768 mg. $\left.\mathrm{g}^{-1}\right)$ and IB (1.696 mg. $\left.\mathrm{g}^{-1}\right)$. As previously described, these values represent the maximum pollutant that can be retained in $1 \mathrm{~g}$ adsorbent (GGRP). The preparation of the isotherms and the calculation of the MAC values were performed following literature recommendations (Pereira et al., 2003; Maghri et al., 2012). The MAC values obtained in this work can be considered satisfactory and suggest GGRP as another alternative in the treat ment of effluents contaminated with CR and IB. Obviously, GGRP can not be suggested as a filter for water treatment plants, since said adsorbent still needs to be evaluated for the removal of several other types of pollutants. Others works using different natural adsorbents for the removal of organic pollutants in aqueous medium also revealed satisfactory MAC values as described in Table 1 


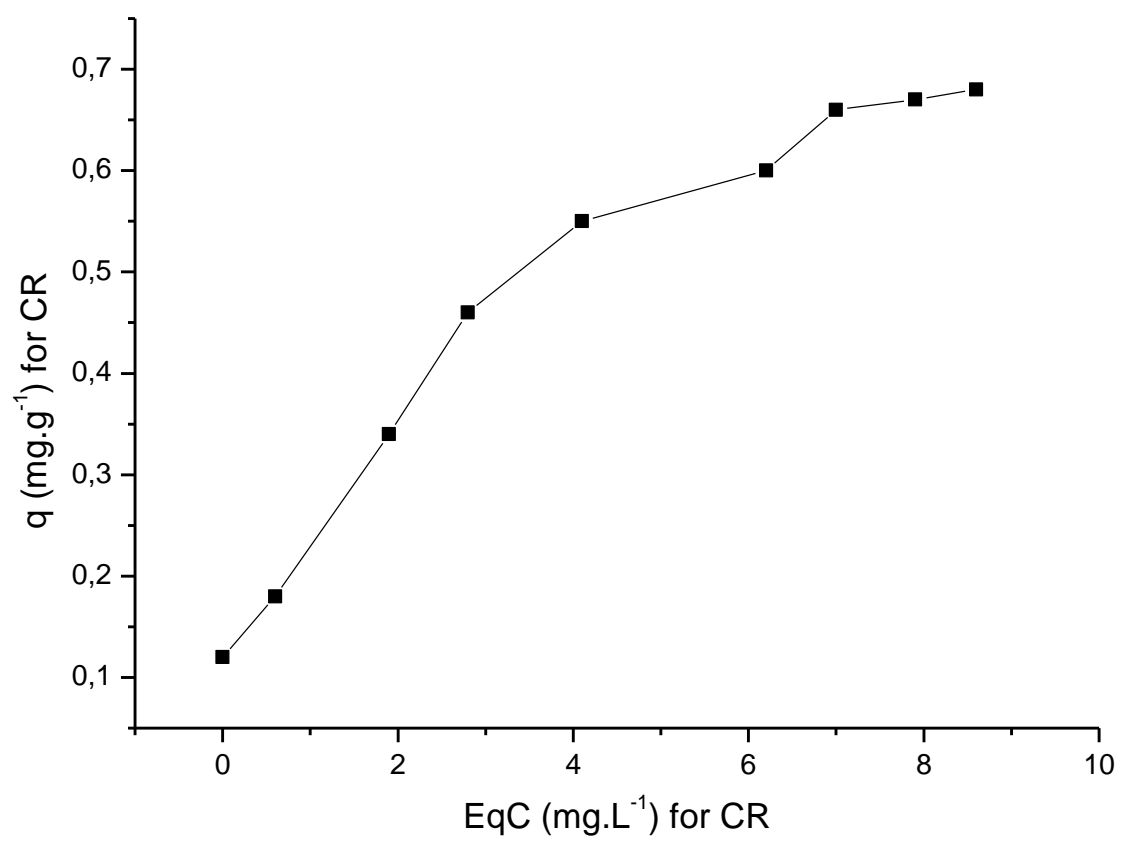

Figure 11:- Adsorption is otherm for CR

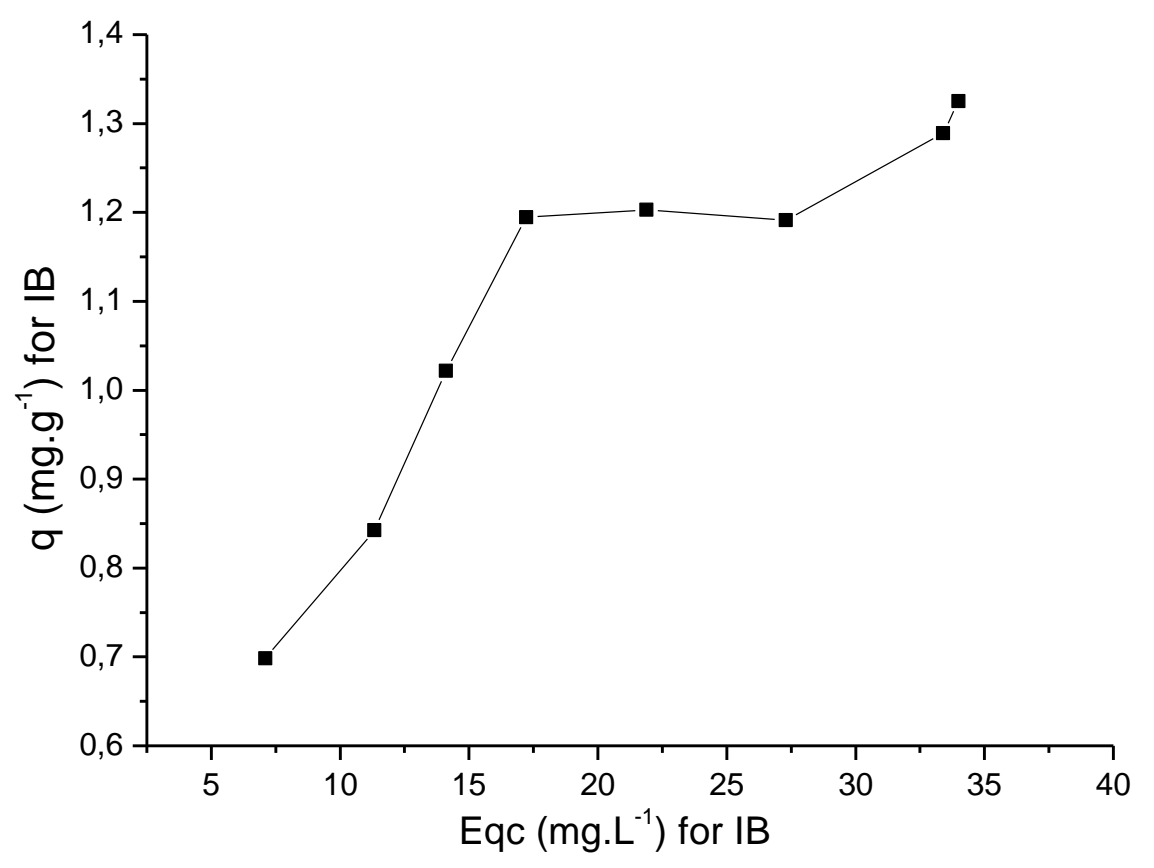

Figure 12:- Adsorption is otherm for IB 


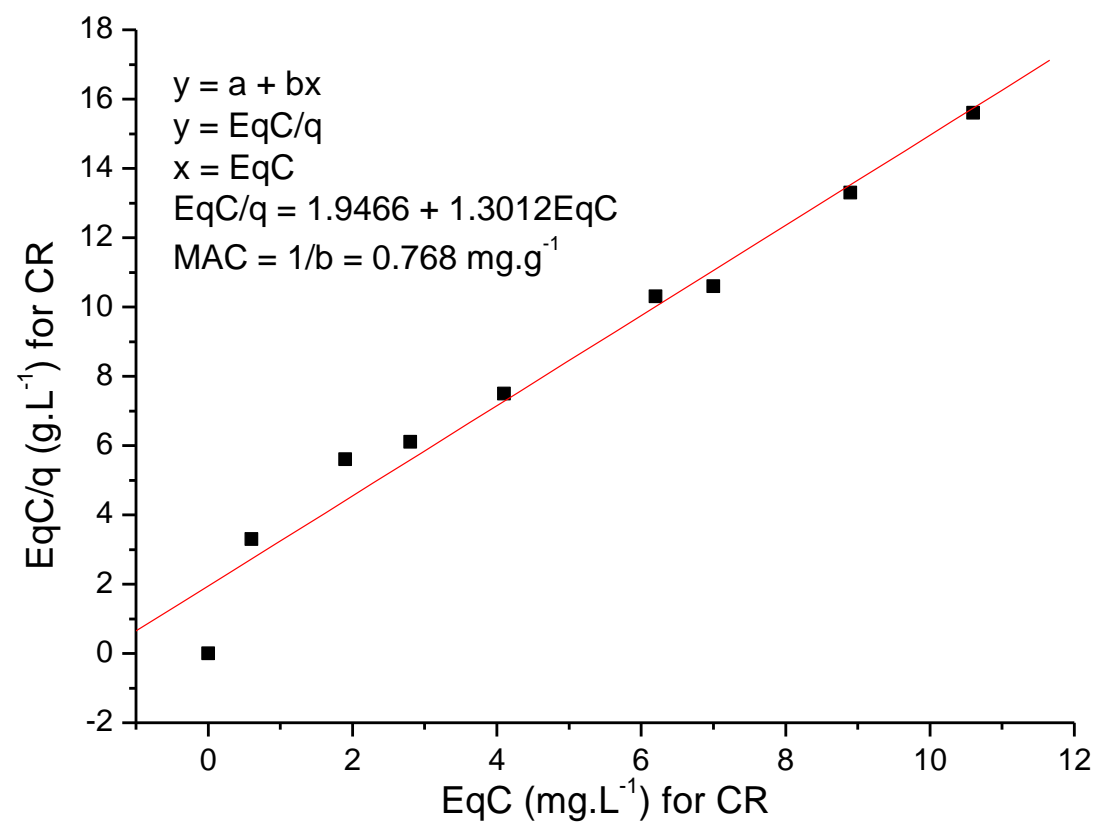

Figure 13:- Adsorption is otherms for $\mathrm{CR}$ after linearization.

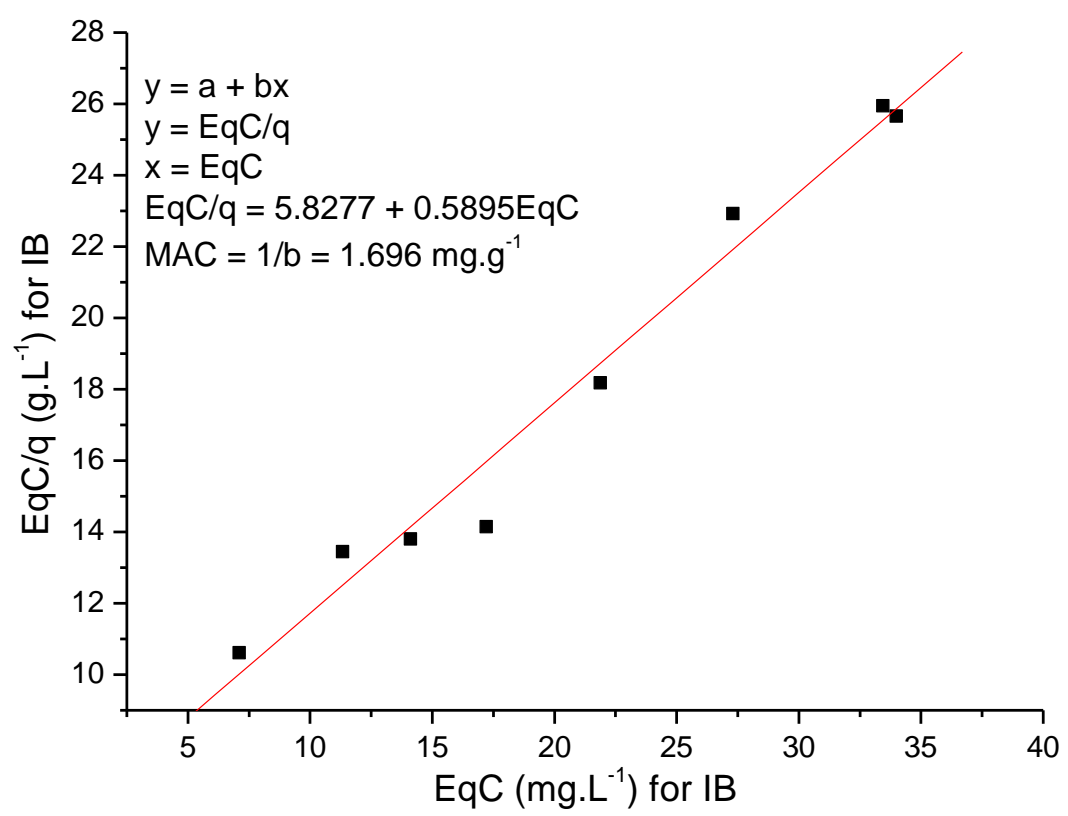

Figure 14:- Adsorption is otherms for IB after linearization.

Table 1:- Different MAC values for different natural adsorbents and organic pollutants

\begin{tabular}{|l|l|l|l|}
\hline Adsorbent & Pollutant & MAC val ues $\mathbf{( m g / g )}$ & References \\
\hline Red mud & Congo red & 4.050 & Namasivayam\&Arasi, 1997 \\
\hline Sugarcane bagasse & Congo red & 4.430 & Ray mundo et al., 2010 \\
\hline
\end{tabular}




\begin{tabular}{|l|l|l|l|}
\hline Sugarcane bagasse & Tetracycline & 0.310 & Ribeiro et al., 2014 \\
\hline $\begin{array}{l}\text { Wood sawdust } \\
\text { powder from Eucalyptus }\end{array}$ & Congo red & 0.523 & Ribeiro et al., 2018 \\
\hline Green coconut mesocarp & Tetracycline & 1.610 & Ribeiro et al., 2016 \\
\hline Green coconut mesocarp & Paracetamol & 0.110 & Ribeiro et al., 2016 \\
\hline Banana peel & Acetylsalicylic acid & 2.290 & Ribeiro et al., 2016 \\
\hline Vermicompost & Crystal violet & 0.780 & Pereira et al., 2009 \\
\hline MytilusEdulis Shells & methylene blue & 3.430 & Maghri et al., 2012 \\
\hline
\end{tabular}

Dyes removal in glass columns:-

Experiments using glass columns containing activated carbon and gravel in the presence and absence of GGRP demonstrated that the percentages of adsorption for both IB and CR are higher in the presence of GGRP than in the presence of activated carbon alone. Table 2 shows the values of the adsorption percentag es for both dyes in the presence and absence of GGRP. In another work (Raymundo et al., 2010) using sugarcane bagasse, the authors demonstrated that this natural adsorbent was very efficient in the removal of CR in effluent $(94 \pm 5 \%)$ enriched with this pollutant.

Table 2: Adsorption of CR and IB in the absence and presence of GGRP

\begin{tabular}{|l|l|l|}
\hline System & IB adsorption $(\%)$ & CR adsorption (\%) \\
\hline AC + Gravel & $13.5 \pm 2.1$ & $16.9 \pm 3.2$ \\
\hline AC + Gravel + GGRP & $98.2 \pm 1.2$ & $82.1 \pm 3.5$ \\
\hline
\end{tabular}

\section{Conclusion:-}

The values of maximum adsorptive capacities and mainly the percentage of retention of the dyes in the columns can be considered satisfactory. The results of the experiments show that GGRP may possibly be used as a further alternative in the treatment of textile effluents containing CR and IB. However, more detailed studies will be needed to make GGRP a viable alternative filter. These studies will include, for example, the evaluation of GGRP as agent to remove other pollutants in aqueous medium and chemical composition of the filtrates after percolation in columns.

\section{Acknowlegdme nts:-}

The authors would like to thank the Federal Institute of Espírito Santo (Vitória, ES, Brazil) by financial support and Laboratory of Cellular Ultra structure Carlos Alberto Redins of Federal University of Espírito Santo (Vitória, ES, Brazil) for the use of scanning electron microscope.

\section{References:-}

1. Adedayo, O., Javadpour, S., Taylor, C., Anderson, W.A., Moo-Young M. Decolourization and detoxification of methyl red by aerobic bacteria from a wastewater treatment plant. World Journal of Microbiology \& Biotechnology, 20, 545-550, 2004.

2. Amin, M.M., Bina, B., Rahimi, A., Heidari, M. Adsorption of gas-phase n-hexane and benzene, toluene, ethyl benzene, and xylene onto compost; kinetics and isothermal studies. International Journal of Environmental Health Engineering, 4, 2, 1-8, 2015.

3. Baig, G.A. A study on the exhaust dyeing of various synthetic fibreswithindigo, Coloration Technology. 128, 114-120, 2012.

4. Chen, H., Motuzas, J., Martens, W., Costa, J.C.D. Surface and catalytic properties of stable Me(Ba, Ca and $\mathrm{Mg}) \mathrm{SrCoO}$ for the degradation of orange II dye under dark conditions. Applied Surface Science, 450, 292-300, 2018.

5. Duke, J.A. Handbook of Medic inal Herbs. CRC Press, Boca Raton, FA, 1985

6. Farrokhi-Rad, M., Mohammadalipour, M., Shahrabi, T. Electrophoretically deposited halloysite nanotubes coating as the adsorbent for the removal of methylene blue from aqueous solution. Journal of the European Ceramic Society, 38, 3650-3659, 2018.

7. Fenwick, G.R. Liquorice, GlycyrrhizaglabraL. Composition, uses and analysis. Food Chemistry, 38, 119-143, 1990.

8. Forgacs, E., Cserhati, T., Oros, G. Removal of synthetic dyes from wastewaters: a review. Environment International, 30, 953-971, 2004. 
9. Guaratini, C.C.I., Zanoni, M.V.B. Textile dyes. Química Nova, 23, 1, 71-78, 2000.

10. Jordão, C. P., Fernandes, R. B. A., Ribeiro, K. L., Barros, P. M., Fontes, M. P. F., Souza, F. M. P. A. Study on $\mathrm{Al}$ (III) and Fe (II) ions sorption by catlle manure vemico mpost. Water Air Soil Pollution, 210, 51 -61, 2010.

11. Kunz, A., Zamora, P.P., Moraes, S.G., Du rán, N. New tendencies on textile effluent treatment. Quimica Nova, 25, 78-82, 2002.

12. Lutomski, J. Chemie un therapeutischeVerwendungvonSüssholz (GlycyrrhizaglabraL.). Pharmazie in unsererZeit, 12, 49-54, 1983.

13. Maghri, I., Amegrissi, F., Mohamed, E., Kenz, A., Tanane, O., Mohamed, T., Salouhi, M. Comparison of Adsorption of Dye Onto Low-Cost Adsorbents. Global Journal of Science Frontier Research Che mistry, 12, 4, p.1-6, 2012.

14. Mathur, N., Bhatnagar, P., Bakre, P. Assessing mutagenicity of textile dyes from pali (rajasthan) using ames bioassay. Applied Ecology and Environ mental Research, 4, 1, 111-118, 2005.

15. Mohammadi-Moghadam, F., Amin, M.M., Hajian, M.K., Momenbeik, F., Nourmoradi, H., Hatamipour, M.S. Application of Glycyrrhizaglabraroot as a novel adsorbent in the removal of toluene vapors: equilibrium, cinetic, and thermodynamic study. Journal of Environ mental and Public Health, 2013, 1 -7, 2013.

16. Munagapat, V.S., Yarramuthi, V., Kim, Y., Lee, K.M., Kim, D.S. Removal of anionic dyes (Reactive Black 5 and Congo Red) from aqueous solutions using Banana Peel Powder as an adsorbent. Ecotoxicology and Environmental Safety, 148, 601-607, 2018.

17. Namasivayam, C., Arasi, J.S.E. Removal of congoredfromwastewater by adsorption onto wasteredmud. Chemosphere, 34, 401-417, 1997.

18. Pagano, M., Ciannarella, R., Locaputo, V., Mascolo, G., Volpe, A. Oxidation of azo and anthraquinonic dyes by peroxy monosulphate activated by UV light. Journal of Environmental Science and Health, Part A, 53, 4, 393404, 2018.

19. Pereira, M.G., Arruda, M.A.Z. Vermicompost as a natural adsorbent material: characterization and potentialities for cad miu m adsorption. Journal of the Brazilian Chemical Society, 14, 39-47, 2003.

20. Pereira, M.G., Korn, M., Santos, B.B., Ramos, M.G. Vermicompost for tintedorganiccationicdyesretention. Water Air Soil Pollution, 200, 227-235, 2009.

21. Pereira, M.G., Andrade, M.V.A.S., Ornelas, V.C., Almeida, R.A. N., Fontes, M. P. F., Ribeiro, J.N., Ribeiro, A.V. F. N., Santos, A.V., Souza, A.N., Araújo, C.B., Araújo, A.C.B., Onofre, C.R.E., Korn, M.G.A. Assessment of physical-chemicalcharacteristics of water and sedimentsfrom a brazilian tropical estuary: status and environmental implications. The Scientific World Journal, 2012, 1-14, 2012.

22. Ramakrishna, K.R., Viraraghavan, T. Dyere movalusinglowcostadsorbents. Water Science and Technology, 36, 2-3, 189-196, 1997.

23. Ray mundo, A.S., Zanarotto, R., Belisário, Marciela., Pereira, M.G., Ribeiro, J.N., Ribeiro, A.V.F.N. Evaluation of sugar-cane bagasse as bioadsorbent in the wastewatertreatmentcontaminatedwithcarcinogeniccongoreddye. Brazilian Archives of Biology and Technology, 53, 931-938, 2010.

24. Ribeiro, A.V.F.N., Cosmo, P.C., Pereira, M.G., Dalfior, B.M., Gonçalves, G.S., Licinio, M.V.V.J., Endringer, D.C.,Oliveira, J.P., Ribeiro, J.N. Use of sugarcane bagasse for adsorption of tetracycline in aqueous medium. Indian Journal of AppliedResearch, 4, 10-14, 2014.

25. Ribeiro, A.V.F.N., Silva, A.R., Cunha, T.P., Santos, R.T.L., Oliveira, J.P., Pereira, E.V., Licinio, M.V.V.J., Pereira, M.G., Santos, A.V., Ribeiro, J.N. Banana peel for acetylsalicylicacidretention. Journal of Environmental Protection, 7, 1850-1859, 2016.

26. Ribeiro ${ }^{b}$, J.N.; Ribeiro, A.V.F.N., Oliveira, J.P., Leão, R.T., Cunha, T.P..Green mesocarp coconut for treatment of water contaminated with paracetamol and tetracycline. International Journal of Scientific Research, 5, 319323, 2016.

27. Ribeiro, A.V.F.N., Silva, A.R., Pereira, M.G. Licinio, M.V.V.J. Wood sawdustpowderfro mCorymbiacitriodora to congoredtoxicdye adsorption. Indian Journal of Applied Research, 8, 7, 449-451, 2018.

28. SahMoune, M.N., Yeddou, A.R. Potential of sawdustmaterials for the removal of dyes and heavymetals: examination of is otherms and kinetics. Desalination and Water Treatment, 57, 50, 24019-24034, 2016.

29. Tahir, H., Sultan, M., Akhtar, N., Hammed, U., Abid, T. Application of natural and modified sugar cane bagasse for the removal of dye from aqueous solution. Journal of Saudi Che mical Society, 20, 115 -121, 2016

30. Wong, K.C. Review of spectrometric identification of organiccompounds, 8th Edition. Journal of Chemical Education, 92, 10, 1602-1603, 2015. 